\title{
Are Virtual Conferences the Future of Learning in This COVID Era?
}

\author{
Jaideep Malhotra ${ }^{1}$, Pavika Lal ${ }^{2}$, Ruchika Garg ${ }^{3}$, Gangadhar Sahoo ${ }^{4}$ \\ Journal of South Asian Federation of Obstetrics and Gynaecology (2020): 10.5005/jp-journals-10006-1817
}

The year 2020 was not the best, to begin with as it brought with it several travel restrictions and health and safety guidelines due to a very well-known COVID-19 pandemic. In the current scenario, it is more important than ever to maximize communication in the scientific and medical community. In the context of academic meetings and conferences, there is a growing need for a set of guidelines secondary to this immense health crisis, as well as the increasing environmental and economic challenges that are faced by academic and medical conferences.

The role of virtual conferencing is expanding to avoid international travel and cross-border contamination as it is the need of the hour. This resulted in a colossal shift in the way scientists, teachers, intellectuals, and universities interact. Many such meetings and conferences which were to take place in 2020 had to face cancellation or were postponed or were moved to digital platforms as colleges, universities, research institutes, and companies had put non-essential work related to travel on hold. As the novel coronavirus outbreak has shuttered businesses and disrupted billions of lives around the globe, learners have increasingly sought out a way to stay intellectually active making learning a dynamic process even in this situation to disseminate the knowledge to the ingenious crowd through online conferences. ${ }^{1}$

Several institutions and organizations, instead of scrapping planned meetings/conferences altogether, switched to virtual platforms. This transition to digital conferencing and virtual webinars was embraced enthusiastically world wide by every scholar even though many had limited if any experience on that front. On the contrary, individuals from the information technology sector who already have been practising virtual conferences and webinars have found a multi-fold increase in the same. Smaller, more focused conferences planned by such organizations also made the switch to an online environment a success story.

\section{Benefits of Webinar \\ Ecofriendly Way}

One of the greatest beneficiaries of the online migration of conferences has been the environment. A recent review estimated that the amount of carbon dioxide generated by each researcher through conference travel ranges from 0.5 to 2 metric tons. Staggeringly, the total carbon footprint of the world's estimated 7.8 million researchers each traveling to one conference a year is equivalent to that of some small nations. ${ }^{2}$

\section{Leveling the Field}

Beyond enabling the participation of scientists/teachers/students who had to cancel their specific travel plans, the move to digital format has democratized attendance at the conference by making
${ }^{1}$ Department of Obstetrics and Gynecology, Rainbow IVF, Agra, Uttar Pradesh, India

${ }^{2}$ Department of Obstetrics and Gynecology, Ganesh Shankar Vidyarthi Memorial Medical College, Kanpur, Uttar Pradesh, India

${ }^{3}$ Department of Obstetrics and Gynaecology, SN Medical College, Agra, Uttar Pradesh, India

${ }^{4}$ Institute of Medical Sciences and SUM Hospital, SOA (Deemed to be University), Bhubaneswar, Odisha, India

Corresponding Author: Ruchika Garg, Department of Obstetrics and Gynaecology, SN Medical College, Agra, Uttar Pradesh, India, Phone: +91 9720004485, e-mail: Ruchikagargagra@gmail.com

How to cite this article: Malhotra J, Lal P, Garg R, et al. Are Virtual Conferences the Future of Learning in This COVID Era? J South Asian Feder Obst Gynae 2020;12(5):269-270.

Source of support: Nil

Conflict of interest: None

them more accessible to a wider and more diverse group of scholars worldwide. Since a virtual conference does not require traveling and therefore it is a more feasible option for whom traveling might be more complicated or infeasible due to visa requirement, pressing family obligations and caregiving responsibilities or disabilities as well as jet lag and motion sickness. ${ }^{3}$

\section{Behind the Scenes}

Reduced cost associated with virtual participation is like a cherry on the cake. Not only it reduces the huge financial burden on organizers (such as, cost incurred in booking venue, providing hospitality) but also permits the registration of attendees who have limited financial means or lives far from the areas that are traditional conference hubs. ${ }^{4}$ Such conferences and webinars also provide flexibility to its consumers who can stream a recorded version mostly at the time of their will. In addition, the opportunity offered by attending a conference from the comfort of one's home may permit more focused and productive participation along with reduced anxiety, especially for larger meetings that might feel more chaotic in-person. One of the major advantages of a webinar is that there is no tie to any particular time zone and all the scientific sessions are available to watch on-demand due to the recording facility. ${ }^{5}$

Despite providing so many advantages over physical meetings/ conferences, this concept of virtual webinars is not all "Hunky Dory". It is easy to see why people do not love the idea of virtual conferences. Sitting for hours behind a computer screen, listening to a floating head, is not exactly a thrilling prospect. We do not get to bask in the exotic setting of a tropical island or other international location. And virtual conferences do not allow researchers to 
retreat from their everyday routines and fully immerse themselves in sharing knowledge. The lack of in-person interaction is also a problem. It is no exaggeration that many a fruitful collaboration and career move can be traced back to a long conference lunch queue. Even being invited to present at a virtual conference can ring somewhat hollow. Alone in the office, speakers must struggle not only with technological demands but also with connecting with their remote audience.

There is no doubt that virtual conferences in the current time of COVID-19 raise challenges for organizers, attendees, and speakers but they have made lemonade out of lemons by moving to online platforms.

\section{VERDICT}

Ultimately, in-person and virtual conferences are not interchangeable. However, the two formats need not be mutually exclusive, and conferences of the future should aim to capture the main strengths of both.

As we all know humans are social beings who thrive on physical interactions. However, that does not discount the success and wide accessibility of digital conferencing. With the pandemic still unabated, it is hard to envision an exclusive return to physical conferences due to their ability to act as a super-spreader event for the COVID-19 pandemic. Perhaps then 2020 will be the year when the unwelcome push of COVID-19 has been a blessing in disguise which spurred us to rethink how to run our conferences/ communication and seek a more inclusive and sustainable mode of information exchange for the future, in the form of "virtual conferences".

\section{References}

1. https://hbr.org/2020/03/what-it-takes-to-run-a-great-virtualmeeting.

2. https://www.scientificamerican.com/article/the-surprisingadvantages-of-virtual-conferences.

3. https://www.nature.com/articles/s43018-020-00108-1.

4. Spiers V. Reflections on the upsurge of virtual cancer conferences during the COVID-19 pandemic. Br J Cancer 2020;123:698-699. DOI: 10.1038/s41416-020-1000-x.

5. https://physicsworld.com/a/the-virtual-conference-thoughts-forma-first-time-attendee/. 